\title{
Sistemas de Liberação Controlada com Bupivacaína Racêmica (S50-R50) e Mistura Enantiomérica de Bupivacaína (S75-R25): Efeitos da Complexação com Ciclodextrinas no Bloqueio do Nervo Ciático em Camundongos *
}

\section{Drug-Delivery Systems for Racemic Bupivacaine (S50-R50) and Bupivacaine Enantiomeric Mixture (S75-R25): Cyclodextrins Complexation Effects on Sciatic Nerve Blockade in Mice}

Daniele Ribeiro de Araújo ${ }^{1}$; Leonardo Fernandes Fraceto ${ }^{2}$; Angélica de Fátima de Assunção Braga, TSA ${ }^{3}$; Eneida de Paula ${ }^{4}$

\section{RESUMO}

Araujo DR, Fraceto LF, Braga AFA, Paula E - Sistemas de Liberação Controlada com Bupivacaína Racêmica (S50-R50) e Mistura Enantiomérica de Bupivacaína (S75-R25): Efeitos da Complexação com Ciclodextrinas no Bloqueio do Nervo Ciático em Camundongos

JUSTIFICATIVA E OBJETIVOS: Os efeitos adversos associados ao uso de bupivacaína levaram à procura por novos anestésicos locais ( $A L$ ) com perfil de bloqueio semelhante e menos tóxicos, surgindo novas preparações como a mistura enantiomérica de bupivacaína (S75-R25). Os sistemas de liberação controlada, contendo AL em carreadores como ciclodextrinas (CD), têm como objetivo melhorar a eficácia anestésica e o índice terapêutico dessas drogas. Este estudo visou a preparação, a caracterização e a avaliação da eficácia anestésica dos complexos de inclusão da mistura enantiomérica da bupivacaína (S75-R25) e da bupivacaína racêmica (S50-R50) com hidroxipropil/-ciclodextrina (HP $\beta-C D)$ comparando-os com as preparações atualmente utilizadas na clínica.

MÉTODO: Os complexos de inclusão foram preparados misturando-se quantidades apropriadas de HPß-CD e S50-R50 ou S75-R25 nas razões molares (1:1 e 1:2) e caracterizados por estudos de solubilidade de fases. Determinaram-se as constantes de afinidade $(K)$ de cada AL pela HPB-CD. Os bloqueios motor e sensorial induzidos pelas drogas livres e complexadas foram avaliados, em camundongos, através do bloqueio do nervo ciático. Para a realização dos experimentos, utilizaram-se três concentrações de AL: 0,125\%;0,25\% e $0,5 \%$

RESULTADOS: Os estudos de solubilidade indicaram a formação de complexos de inclusão de S50-R50 e S75-R25 com HP $\beta-C D$, com valores de constante de afinidade (K) similares para os dois anestésicos: $14,7 \mathrm{M}^{-1}$ (S50-R50:HP- $\beta C D$ ) e 14,3 $\mathrm{M}^{-1}$ (S75-R25:HP- $\beta C D$ ). Os testes em animais mostraram que a complexação potencializou o bloqueio nervoso diferencial induzido pelos $A L: i)$ a duração do bloqueio motor induzido por S75-R25 foi similar à do S50-R50, mas menos intenso $(p<0,001)$. Já os complexos, S50-R50HP $\beta-C D$ e $S 75-R 25 H P \beta-C D$ reduziram a latência ( $p<0,01$ e $p<0,05$, respectivamente) sem modificar

* Recebido do (Received from) Laboratório de Biomembranas e Sistemas Biomiméticos, Departamento de Bioquímica - Instituto de Biologia, Universidade Estadual de Campinas - UNICAMP

1. Doutoranda em Biologia Funcional e Molecular - Bioquímica da UNICAMP

2. Professor Doutor da Faculdade de Farmácia e Bioquímica, Universidade de Sorocaba - UNISO

3. Professora Associada do Departamento de Anestesiologia - Faculdade de Ciências Médicas da UNICAMP

4. Professora Associada do Departamento de Bioquímica, Instituto de Biologia da UNICAMP

Apresentado (Submitted) em 21 de setembro de 2004 Aceito (Accepted) para publicação em 31 de janeiro de 2005

Endereço para correspondência (Correspondence to)

Dra. Daniele Ribeiro de Araújo

Universidade Estadual de Campinas - UNICAMP

Cidade Universitária Zeferino Vaz $s / n$

Depto. de Bioquímica- Instituto de Biologia

13083-970 Campinas, SP

E-mail:draraujo2003@yahoo.com.br

(c) Sociedade Brasileira de Anestesiologia, 2005 intensidade do bloqueio motor ( $\left.E_{m a ́ x}\right)$, em relação às drogas livres; ii) a avaliação do bloqueio sensorial mostrou aumento na intensidade da analgesia com os sistemas S50-R50HP $\beta-C D(2$ vezes, $p<0,001)$ e S75-R25HP $\beta-C D(1,5-1,8$ vezes; $p<0,01$ e $p<0,001$, respectivamente), nas duas proporções molares (1:1 e 1:2, $A L: C D)$, além do prolongamento da duração do efeito analgésico, quando comparados às formulações S50-R50 e S75-R25. CONCLUSÕES: Os efeitos analgésicos mais pronunciados, obtidos após a complexação

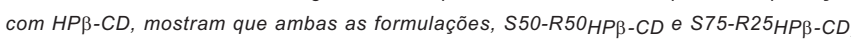
são de grande interesse para o alivio da dor no periodo pós-operatório, com a vantagem de serem administradas em menores concentrações. Entretanto, há que se ressaltar que a mistura enantiomérica de bupivacaína (S75-R25), por ser menos tóxica, representa uma alternativa no desenvolvimento de formulações de liberação controlada mais seguras $e$ eficazes do que com a bupivacaína racêmica (S50-R50).

Unitermos: ANESTÉSICOS, Local: bupivacaína; ANIMAIS, camundongos

\section{SUMMARY}

Araujo DR, Fraceto LF, Braga AFA, Paula E - Drug-Delivery Systems for Racemic Bupivacaine (S50-R50) and Bupivacaine Enantiomeric Mixture (S75-R25): Cyclodextrins Complexation Effects on Sciatic Nerve Blockade in Mice

BACKGROUND AND OBJECTIVES: Bupivacaine-induced side effects have led to the search for new local anesthetics (LA) with similar potency and lower toxicity, such as bupivacaine enantiomeric mixture (S75-R25). Drug-delivery systems for LA in carriers, such as cyclodextrins $(C D)$, have been developed to improve anesthetic potency and therapeutic index of those drugs. This study aimed at preparing, characterizing and evaluating the anesthetic efficacy of inclusion complexes of bupivacaine enantiomeric mixture (S75-R25) and racemic bupivacaine (S50-R50) with hydroxypropy/ $\beta$ - cyclodextrin (HP $\beta-C D)$ comparing them to clinically available preparations.

METHODS: Inclusion complexes were obtained by mixing appropriate volumes of HP $\beta-C D$ and S50-R50 or S75-R25 to final 1:1 or 1:2 molar ratios and were characterized by phase solubility experiments. Affinity constants $(K)$ were determined between $H P \beta-C D$ and each $L A$. Motor and sensory blocks induced by plain or complexed $L A$ formulations were evaluated in mice by sciatic nerve block. Three $L A$ concentrations were used during the experiment: $0.125,0.25$ and $0.5 \%$. RESULTS: Solubility experiments results were indicative of S50-R50:HP $\beta-C D$ and S75-R25:HP $\beta-C D$ complexation, with similar affinity constant $(K)$ values: $14.7 \mathrm{M}^{-1}$ and $14,3 \mathrm{M}^{-1}$, respectively. In vivo experiments have shown that complexation has enhanced differential nerve blockade induced by LA: i) motor blockade duration induced by S75-R25 was similar, to the induced by but less intense S50-R50 ( $p<0.001)$. S50-R50HP $\beta-C D$ and S75-R25HP $\beta-C D$ complexes have decreased onset ( $p<0.01$ and $p<0.05$, respectively), without changing motor block intensity $\left(E_{\max }\right)$ as compared to plain drugs; ii) sensory block evaluation has revealed higher analgesic intensity with S50-R50HPß-CD (2-fold, $p$ $<0.001)$ and S75-R25HPß-CD (1.5-1.8-fold, $p<0.01$ and $p<0.001$, respectively) with both molar ratios (1:1 and 1:2, LA:CD), in addition to prolonging analgesic effect as compared to S5O-R50 and S75-R25.

CONCLUSIONS: More pronounced analgesic effects obtained by complexation with

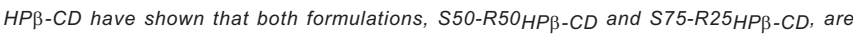
very useful for postoperative pain relief, requiring lower LA concentrations. Nevertheless, it is worth noticing that S75-R25 - being less toxic than racemic bupivacaine - is an interesting alternative for the development of more effective and safe drug-delivery systems as compared to racemic bupivacaine (S50-R50).

Key Words: ANESTHETICS, Local: bupivacaine; ANIMALS, mices 


\section{INTRODUÇÃO}

A bupivacaína é o anestésico local $(A L)$ pertencente à classe das amino-amidas, mais utilizado em procedimentos cirúrgicos, principalmente para anestesias regionais prolongadas. Os efeitos adversos (toxicidade para os sistemas nervoso central e cardiovascular) associados ao uso de bupivacaína levaram à pesquisa de novos agentes com perfil de bloqueio semelhante, mas com menor cardiotoxicidade ${ }^{1}$. Da pesquisa resultaram AL como a ropivacaína e a levobupivacaína - sintetizados sob a forma enantiomérica $\mathrm{S}(-)$ - demonstrando que, além de modificações moleculares, a estereosseletividade é fator importante para diminuir a cardiotoxicidade da bupivacaína. Assim, estudos recentes mostraram uma redução na capacidade disritmogênica e no risco de convulsões induzidas por levobupivacaína ${ }^{2-3}$, bem como a manutenção de propriedades anestésicas similares à bupivacaína ${ }^{4}$. $O$ advento das pesquisas sobre estereosseletividade possibilitou a modificação das proporções dos estereoisômeros $\mathrm{R}(+)$ e $\mathrm{S}(-)$ da bupivacaína e a síntese de nova formulação anestésica local, contendo $25 \%$ do isômero $R(+)$-bupivacaína e $75 \%$ do isômero S(-)-bupivacaína, melhorando o perfil anestésico da droga em relação à levobupivacaína e aumentando sua margem de segurança ${ }^{5}$.

Embora existam diferenças quanto aos perfis farmacocinético e farmacodinâmico dos enantiômeros, propriedades físico-químicas como a solubilidade aquosa ou em lipídios e o pKa são preservadas ${ }^{4}$ constituindo-se em fatores que podem limitar o benefício terapêutico de drogas administradas na forma tradicional. Uma alternativa utilizada é o desenvolvimento de sistemas de liberação controlada contendo $\mathrm{AL}$ em carreadores, como ciclodextrinas (CD), que possibilitam a manipulação de algumas propriedades físico-químicas além de melhorarem os efeitos terapêuticos, favorecendo sua utilização clínica ${ }^{6}$.

As CD são oligossacarídeos cíclicos, constituídos por unidades de glicose (Figura 1), capazes de complexarem em sua cavidade hidrofóbica moléculas de tamanho e polaridade apropriados, formando complexos de inclusão. A complexação altera as propriedades físico-químicas, como a solubilidade, estabilidade e biodisponibilidade das drogas ${ }^{7}$. De fato, essa propriedade das CD torna bastante promissor seu uso para a complexação de $A L$, e diversos estudos utilizando bupivacaína racêmica têm demonstrado aumento na duração de ação e uma melhora significante no seu índice terapêutico 8-11. Dessa forma, torna-se interessante verificar se a complexação com CD alteraria também o perfil anestésico da mistura enantiomérica de bupivacaína, uma formulação potencialmente menos tóxica, em relação à bupivacaína racêmica. Este estudo teve por objetivo preparar, caracterizar e avaliar, em camundongos, a eficácia anestésica dos complexos de inclusão com hidroxipropil $\beta$-ciclodextrina (HP $\beta-C D$, Figura 1) com a mistura enantiomérica da bupivacaína (S75-R25) ou da bupivacaína racêmica (S50-R50) e compará-los com as formulações atualmente utilizadas na clínica.

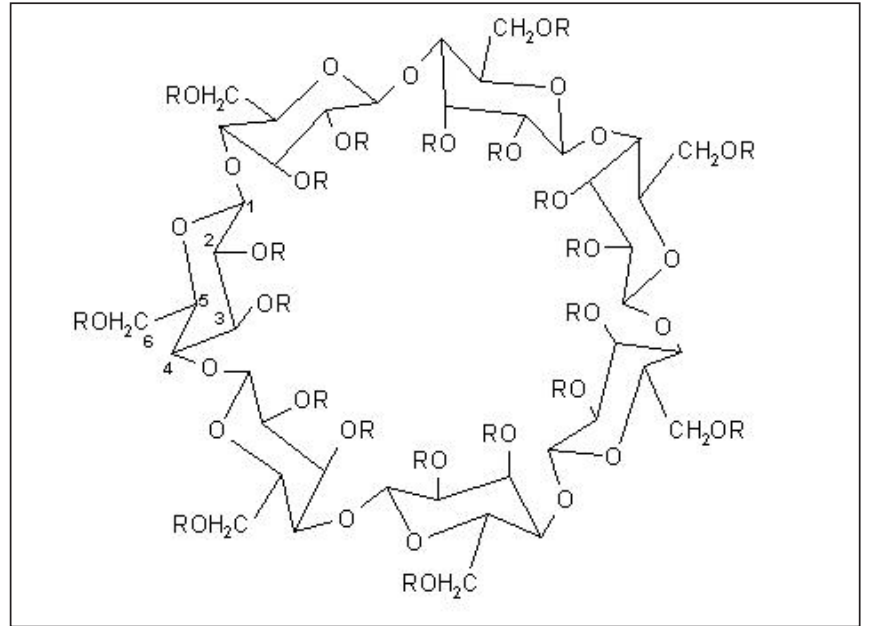

Figura 1 - Estrutura Química da $\beta-C D$, com 7 Monômeros de Glicose e $\mathrm{R}=\mathrm{H}$. R $=\mathrm{CH}_{2} \mathrm{CHOHCH}_{3}$ ou H, no Caso da HP $\beta-\mathrm{CD}$ (substituições nos carbonos 2, 3 e 5)

\section{MÉTODO}

Os complexos sólidos foram preparados misturando-se quantidades apropriadas de HP $\beta$-CD e S50-R50 ou S75-R25 em água, de maneira que a razão molar fosse 1:1 ou 1:2. As amostras foram deixadas sob agitação durante 24 horas à temperatura ambiente. Após isso, a solução foi liofilizada e guardada $-20^{\circ} \mathrm{C}$ para posterior uso ${ }^{9}$. Os complexos sólidos foram pesados em quantidades suficientes para obter as concentrações de drogas desejadas e, posteriormente, dissolvidos em tampão (HEPES 20mM pH 7,4, com NaCl 154 $\mathrm{mM})$. As concentrações de S50-R50 e S75-R25 utilizadas foram: $0,125 \% ; 0,25 \%$ e $0,5 \%$.

Para a caracterização físico-química dos complexos formados, realizaram-se experimentos de solubilidade de fases ${ }^{12}$ determinando-se as constantes de afinidade (K) pela HP $\beta-C D$, para cada AL. Os experimentos foram realizados para avaliar a solubilidade dos dois $\mathrm{ALem} \mathrm{pH} 7,4$, sendo que na ausência de HP $\beta$-CD - tanto a S50-R50 quanto a S75-R25 são insolúveis na concentração utilizada (30 mM). Neste ensaio, concentrações crescentes de $\operatorname{HP} \beta-C D(0,5,10$ e 20 $\mathrm{mM}$ ) foram adicionadas a $30 \mathrm{mM}$ de S50-R50 ou S75-R25 e, posteriormente, dissolvidas em solução tampão (HEPES 50 $\mathrm{mM}, \mathrm{pH} 7,4, \mathrm{com} \mathrm{NaCl} 154 \mathrm{mM}$ ). As amostras foram agitadas à temperatura ambiente durante 24 horas, até atingirem o equilíbrio. Após esse período, as amostras foram filtradas e analisadas por espectrofotometria (espectrofotômetro Beckman DU-70) a um comprimento de onda de $260 \mathrm{~nm}^{13}$, calculando-se o valor da concentração de AL na solução. Todos os experimentos foram realizados em triplicatas. A alta razão molar AL:HP $\beta-C D$ (até 2:1) utilizada nestes ensaios é justificada pelo fato de a constante de afinidade (K) ser calculada utilizando-se a porção linear do diagrama de solubilidade de fases, de acordo com a equação ${ }^{12}$ : 
$\mathrm{K}=$

Inclinação da Reta

Solubilidade Inicial do AL (1-Inclinação da reta)

\section{Avaliação da Eficácia Anestésica}

Este estudo experimental foi realizado após aprovação pela Comissão de Ética na Experimentação Animal do Instituto de Biologia da Universidade Estadual de Campinas (CEEA) - IB - UNICAMP que segue as normas do Colégio Brasileiro de Experimentação Animal (COBEA). Foram utilizados grupos de 7 camundongos albinos, machos, pertencentes à linhagem Swiss, com idade aproximada de 60 dias e massa corporal variando entre 30 e 35 gramas. Os animais, provenientes do Centro de Bioterismo da UNICAMP (CEMIB), foram submetidos a ciclo claro/escuro de 12 horas, com água e alimentação à vontade, alojados coletivamente ( 5 animais por gaiola) e aclimatados ao local de experimentação por pelo menos sete dias.

Os ensaios de eficácia anestésica foram realizados pela técnica do bloqueio do nervo ciático em camundongos ${ }^{14}$. As formulações, inclusive o veículo, foram administradas em ordem aleatória por infiltração $(0,1 \mathrm{~mL})$ no espaço popliteal localizado na superfície posterior do joelho, na região do nervo ciático. A avaliação dos bloqueios motor e sensorial foi realizada simultaneamente e todas as medidas foram executadas pelo mesmo investigador. Os animais foram observados durante 24 horas após o tratamento para verificar a incidência de efeitos tóxicos sistêmicos (convulsões e óbitos) ou locais (falhas na recuperação dos movimentos normais na pata injetada). Os grupos experimentais foram assim constituídos:

- Grupo Controle: HP $\beta-C D$

- Grupo S50-R50: bupivacaína racêmica

- Grupo S75-R25: mistura enantiomérica de bupivacaína

- Grupo S50-R50 HPß-CD (1:1): bupivacaína racêmica com hidroxipropilß-ciclodextrina na proporção molar 1:1 (S50-R50:HP $\beta-C D)$

- Grupo S50-R50 HPß-CD (1:2): bupivacaína racêmica com hidroxipropilß-ciclodextrina na proporção molar 1:2 (S50-R50:HP $\beta-C D)$

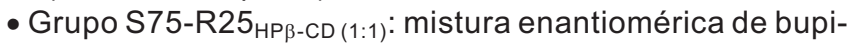
vacaína com hidroxipropil $\beta$-ciclodextrina, na proporção molar 1:1 (S75-R25:HP $\beta$-CD)

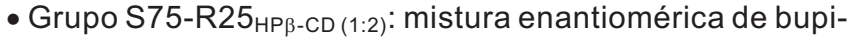
vacaína com hidroxipropil $\beta$-ciclodextrina, na proporção molar 1:2 (S75-R25:HP $\beta-C D)$

Para a avaliação do bloqueio motor, antes da experiência mediu-se a habilidade de cada camundongo em caminhar normalmente, com os quatro membros, por uma tela metálica (com orifícios de $5 \mathrm{~mm}$ de diâmetro) na posição normal e invertida. Somente os animais que preencheram tal requisito foram submetidos à experimentação. O grau de bloqueio motor foi avaliado de acordo com os valores de escore: 0 (movimentação normal), 1 (incapacidade de flexionar completa- mente o membro injetado)e 2 (paralisia total do membro injetado) nos seguintes momentos: a cada minuto durante os 5 primeiros minutos após a administração e, posteriormente, em intervalos de 10 minutos até que fosse verificada a total recuperação dos movimentos do animal ${ }^{15}$. Alatência (tempo entre a injeção e a obtenção de bloqueio motor grau 1 ou 2), o tempo para atingir o escore máximo ( $\left.T_{\text {máx }}\right)$, o valor de escore máximo (efeito máximo, $E_{\text {máx }}$ ), o tempo para recuperação da função motora e o efeito total do anestésico local (estimado pela área sobre a curva efeito-tempo, AUC) foram os parâmetros analisados.

O bloqueio sensorial foi medido utilizando-se um estímulo mecânico ${ }^{16}$. Para a execução do teste utilizou-se equipamento apropriado, chamado analgesímetro, que gera aumento gradual da força (em gramas) sobre a superfície dorsal da pata do animal. Para evitar analgesia induzida por estresse, cada animal foi envolvido em uma toalha permitindo que a cabeça e os membros ficassem livres. O reflexo de retirada da pata foi considerado representativo do limiar de dor (Pain Withdrawal Threshold to Pressure-PWTP). Alinha basal do PWTP foi medida antes dos experimentos e os animais que apresentaram valor do limiar de dor abaixo ou acima de 30 a $50 \mathrm{~g}$ foram excluídos do experimento e estabeleceu-se um valor máximo de pressão (cut-off) de $150 \mathrm{~g}$, considerado representativo de bloqueio sensitivo. Após administração do veículo ou das drogas, as medidas foram realizadas durante 5 horas, com intervalos de 15 minutos durante a primeira hora, 30 minutos durante a segunda e terceira horas e, finalmente, 60 minutos nas últimas duas horas de teste. Aanalgesia foi definida como aumento no limiar de pressão tolerado pelos animais, de no mínimo $50 \%$ maior que o observado no grupo tratado com o veículo. O ponto final da analgesia foi estabelecido quando da ausência de diferença estatística entre os grupos teste e controle ${ }^{17}$.

A análise estatística para a avaliação do bloqueio motor (latência, $T_{\text {máx }}$, tempo de recuperação, $E_{\text {máx }}$ e AUC) foi executada utilizando o teste de Kruskal-Wallis e os dados foram expressos como medianas (limite mínimo e limite máximo). Os valores de PWTP durante o bloqueio sensorial foram expressos em média \pm desvio padrão (DP) e avaliados, em tempos individuais, por Análise de Variância (One-way ANOVA) com teste posterior de Tukey-Kramer. A significância estatística foi definida como $p<0,05^{18}$.

\section{RESULTADOS}

Caracterização dos Complexos de Inclusão com Ciclodextrinas

Através dos experimentos de solubilidade de fases, foi possível observar a complexação da S50-R50 e S75-R25 com $\mathrm{HP} \beta-\mathrm{CD}$ em pH 7,4 e $25^{\circ} \mathrm{C}$ (Figura 2). O diagrama de solubilidade de fases dos sistemas indica a formação de complexos solúveis, havendo aumento linear na solubilidade de S50-R50 e S75-R25 (cerca da 1,3 vezes) em função das concentrações de HP $\beta-C D$ testadas. Os valores obtidos para as constantes de afinidade $(K)$ foram similares para os dois $A L$, 
sendo $14,7 \mathrm{M}^{-1}$ para S50-R50:HP- $\beta$ CD e $14,3 \mathrm{M}^{-1}$ para S75-R25:HP- $\beta$ CD.

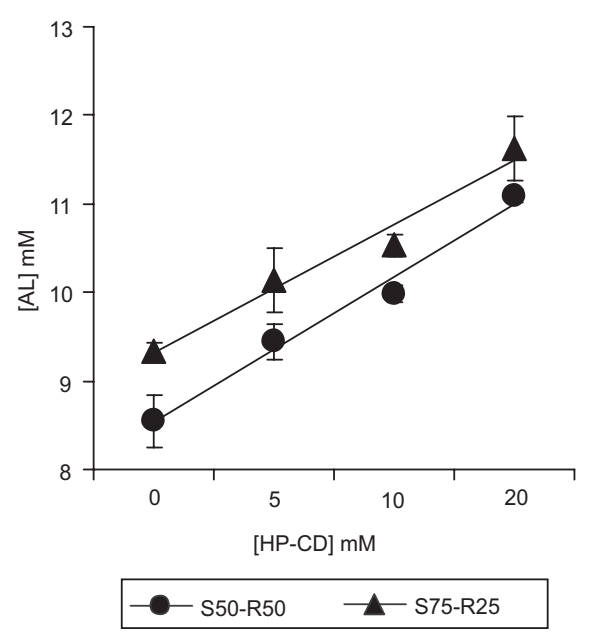

Figura 2 - Curvas de Solubilidade para a S50-R50 e S75-R25 na Presença de Concentrações Crescentes de HP- $\beta C D$, Tampão HEPES $50 \mathrm{mM}, \mathrm{pH} 7,4,25^{\circ} \mathrm{C}$

\section{Avaliação do Bloqueio Motor}

A injeção dos AL livres e complexados alterou, de maneira concentração-dependente, a função motora após o bloqueio do nervo ciático havendo a perda reversível dos reflexos motores em todos os animais tratados (Tabela I). Já o grupo tratado com o veículo (HP- $\beta C D$ ) não apresentou qualquer sinal de bloqueio motor.

Comparações entre as drogas livres (S50-R50 e S75-R25) mostraram que a administração de $\$ 75-\mathrm{R} 25$ induziu redução na latência $(p<0,001)$, em todas as concentrações testadas, embora uma menor intensidade do bloqueio motor tenha sido observada apenas na concentração de 0,125\% ( $p<$ 0,001 ; como evidenciado pelos valores de $E_{\text {máx }}$ ) em relação à S50-R50. No entanto, o tempo de recuperação do bloqueio motor e o efeito total ( $A \cup C$ ) induzido pelas duas preparações comerciais foram similares.

As formulações complexadas contendo S50-R50 induziram diminuição na latência do bloqueio motor em relação à droga livre em todas as concentrações utilizadas $(p<0,01$; para os complexos de inclusão 1:1 e 1:2). Além disso, a análise estatística também revelou diferenças entre S50-R50 ${ }_{H P \beta-C D ~(1: 2)} \mathrm{e}$ S50-R50 para os parâmetros $T_{\text {máx }}(p<0,05)$, tempo de recu-

Tabela I - Latência, Tmáx, Tempo de Recuperação, $E_{\text {máx }}$ e Efeito Total do Bloqueio Motor (AUC) Induzido pelas Formulações de S50-R50 ou S75-R25 Livres e Complexadas com HP $\beta$-CD (1:1 e 1:2) durante o Bloqueio do Nervo Ciático em Camundongos

\begin{tabular}{|c|c|c|c|c|c|c|}
\hline $\begin{array}{l}\text { Concentração } \\
(\%)\end{array}$ & Grupos & $\begin{array}{c}\text { Latência } \\
\text { (seg.) }\end{array}$ & $\begin{array}{c}\mathrm{T}_{\operatorname{máx}} \\
\text { (min.) }\end{array}$ & $\begin{array}{l}\text { Tempo de Recuperação } \\
\text { (min.) }\end{array}$ & $\begin{array}{c}E_{\text {máx }} \\
\text { (escore) }\end{array}$ & $\begin{array}{c}\text { AUC } \\
\text { (escore/h) }\end{array}$ \\
\hline \multirow[t]{6}{*}{$0,125 \%$} & S50-R50 & $60(60-120)$ & $3(2-3)$ & $20(-)$ & $2(-)$ & $22,5(19-28)$ \\
\hline & S50-R50 ${ }_{H P \beta-C D}(1: 1)$ & $30(30-60)^{\mathrm{a}^{* *}}$ & $1(1-2)$ & $30(20-40)$ & $1(1-2)$ & $24(17-36)$ \\
\hline & $\mathrm{S} 50-\mathrm{R} 50_{\mathrm{HP} \beta-\mathrm{CD}(1: 2)}$ & $25(15-35)^{b^{\mathrm{b*t}}}$ & $1(1-4)$ & $40(30-50)^{b^{*}}$ & $1(1-2)$ & $34(24-48)$ \\
\hline & S75-R25 & $40(38-46)^{f \star \star \star *}$ & $1(-)$ & $30(30-50)$ & $1(-)^{f+* t+}$ & $34(24-44)$ \\
\hline & $\mathrm{S} 75-\mathrm{R} 25_{\mathrm{HP} \beta-\mathrm{CD}(1: 1)}$ & $30(25-40)^{c^{*}}$ & $1(1-3)$ & $40(20-60)$ & $1(-)$ & $34(24-54)$ \\
\hline & $\mathrm{S} 75-\mathrm{R} 25_{\mathrm{HP} \beta-\mathrm{CD}(1: 2)}$ & $20(18-25)^{\mathrm{d}^{\star \star}}$ & $2(1-2)$ & $40(30-60)$ & $1(1-2)$ & $47,5(27-54)$ \\
\hline \multirow[t]{6}{*}{$0,25 \%$} & S50-R50 & $60(60-120)$ & $2(2-3)$ & $40(30-40)$ & $2(-)$ & $41(37-58)$ \\
\hline & S50-R50 & $30(30-60)^{\mathrm{a}^{\star \star \star}}$ & $1(1-2)$ & $50(40-60)$ & $2(1-2)$ & $60(44-78)$ \\
\hline & S50-R50 HPß-CD (1:2) & $20(20-30)^{b^{\mathrm{b*}}}$ & $2(1-3)$ & $70(50-80)^{b^{*}}$ & $2(1-2)$ & $67(44-80)^{b^{*}}$ \\
\hline & S75-R25 & $40(38-46)^{f \star \star \star *}$ & $1(-)$ & $30(30-50)$ & $1(-)^{\mathrm{fstat}}$ & $34(24-44)$ \\
\hline & $\mathrm{S} 75-\mathrm{R} 25_{\mathrm{HP} \beta-\mathrm{CD}(1: 1)}$ & $25(20-30)^{c^{*}}$ & $1(-)$ & $60(30-60)$ & $1(1-2)$ & $54(24-54)$ \\
\hline & $\mathrm{S} 75-\mathrm{R} 25_{\mathrm{HP} \beta-\mathrm{CD}(1: 2)}$ & $18(17-22)^{\mathrm{d}^{\star \star}}$ & $2(1-4)$ & $70(40-80)$ & $2(1-2)$ & $74(48-78)$ \\
\hline \multirow[t]{6}{*}{$0,5 \%$} & S50-R50 & $60(-)$ & $1(-)$ & $50(30-60)$ & $2(-)$ & $58(50-88)$ \\
\hline & S50-R50 HРß-CD (1:1) & $30(-)^{\mathrm{a}^{* *}}$ & $1(1-2)$ & $60(50-70)$ & $2(-)$ & $88(78-98)$ \\
\hline & S50-R50 & $15(10-17)^{\mathrm{bt*}}$ & $2(1-3) b^{*}$ & $90(80-100)^{b^{\mathrm{b}+4}}$ & $2(-)$ & $100,5(97,5-108)^{b *}$ \\
\hline & S75-R525 & $40(35-45)^{p+4 x}$ & $1(1-3)$ & $60(40-60)$ & $2(-)$ & $67,5(50,5-68)$ \\
\hline & $\mathrm{S} 75-\mathrm{R} 25_{\mathrm{HP} \beta-\mathrm{CD}(1: 1)}$ & $17(15-25)^{\mathrm{c}^{* *}}$ & $1(1-3)$ & $60(50-60)$ & $2(1-2)$ & $60(49-68)$ \\
\hline & $\mathrm{S} 75-\mathrm{R} 25_{\mathrm{HP} \beta-\mathrm{CD}(1: 2)}$ & $12(10-12)^{\mathrm{d}^{\star \star}}$ & $2(1-2)$ & $90(60-100)^{d^{*}}$ & $2(1-2)$ & $97,5(68-118)^{d, e^{*}}$ \\
\hline
\end{tabular}

Dados expressos em mediana (limite mínimo - limite máximo) ( $\mathrm{n}=7$ /grupo). a S50-R50 ${ }_{\mathrm{HP} \beta-\mathrm{CD}(1: 1)}$ versus S50-R50; b S50-R50 ${ }_{\mathrm{HP} \beta-\mathrm{CD}}(1: 2)$ versus S50-R50; c

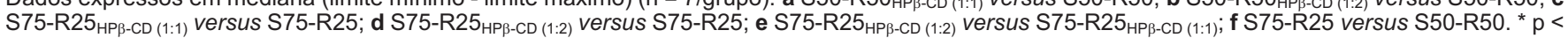
$0,05,{ }^{* *} p<0,01 e^{* * *} p<0,001$ (Teste de Kruskal-Wallis) 
peração e AUC ( $p<0,001$ e $p<0,05$, respectivamente) na concentração de $0,5 \%$.

Com os sistemas S75-R25 HP $-\mathrm{CD}(1: 1$ e 1:2), o tempo de latência foi significativamente menor ( $p<0,05$ e $p<0,01$, respectivamente) quando comparados à formulação livre, nas diferentes concentrações. Em relação à AUC, esta foi significativamente maior $(p<0,05)$ com o complexo na razão molar 1:2 do que após a administração do complexo 1:1 e da formulação livre, na concentração de 0,5\%.

\section{Avaliação do Bloqueio Sensorial}

Aavaliação do bloqueio sensorial, pelo teste PWTP, mostrou relações concentração-dependente para as formulações livres e para os complexos de inclusão de S50-R50 ou S75-R25 com HP $\beta-C D$. Os efeitos do tratamento com $\mathrm{HP} \beta-\mathrm{CD}$, com as drogas livres e complexadas foram avaliados ao longo dos momentos (Figuras 3 e 4).

Ainfiltração do veículo na região do nervo ciático não modificou os valores de linha basal para o limiar da dor em camundongos. Contudo, todas as formulações elevaram o limiar de dor dos animais tratados, que foi estatisticamente diferente daquele do grupo controle ( $p<0,001)$. Além disso, os complexos com HP $\beta-C D$ aumentaram a duração e a intensidade do bloqueio sensorial, quando comparados às drogas livres $(p<0,001)$. No entanto, comparações entre os complexos (1:1 e 1:2, em todas as concentrações) mostraram perfis de bloqueio sensorial semelhantes.

A administração das formulações complexadas, $\mathrm{S} 50-\mathrm{R} 50_{\mathrm{HP} \beta-\mathrm{CD}(1: 1 \text { e 1:2) }}$ modificou a resposta dos animais ao estímulo mecânico no PWTP, aumentando o limiar da dor e a duração do efeito antinociceptivo. A injeção dos complexos na concentração de 0,125 \% induziu aumento na duração da analgesia (em até 4 horas) e na intensidade do efeito (cerca de 2 vezes aos 120 minutos, $p<0,001$ ) quando comparada à da formulação livre (Figura 3A). O tratamento com as formulações complexadas na concentração de 0,25\% também mostrou potencialização do efeito analgésico aos 150 minutos em relação à S50-R50 (2 vezes, $p<0,001$ ) (Figura $3 \mathrm{~B}$ ). Com as formulações complexadas a $0,5 \%$, observou-se perfil semelhante ao apresentado com a concentração intermediária, sendo a intensidade da analgesia significativamente maior $(p<0,001)$ do que aquela observada com a droga livre (Figura $3 \mathrm{C}$ ).

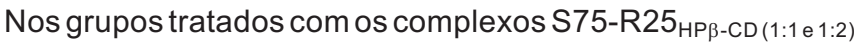
nas diferentes concentrações, a intensidade da analgesia foi cerca de 1,5 a 1,8 vezes maior do que com a droga livre. Além disso, a duração do efeito analgésico foi significativamente maior com as drogas complexadas ( $p<0,001$ nas concentrações de $0,125 \%$ e $0,25 \%$ ) que aquela com a droga livre (240 a 300 minutos x 150 a 300 minutos, respectivamente) (Figuras 4Ae 4B). Na concentração de 0,5\%, embora a duração da analgesia com os complexos tenha sido semelhante à apresentada pela droga livre, o limiar de dor após a injeção dos complexos foi significativamente maior $(p<0,01)$ (Figura 4C).

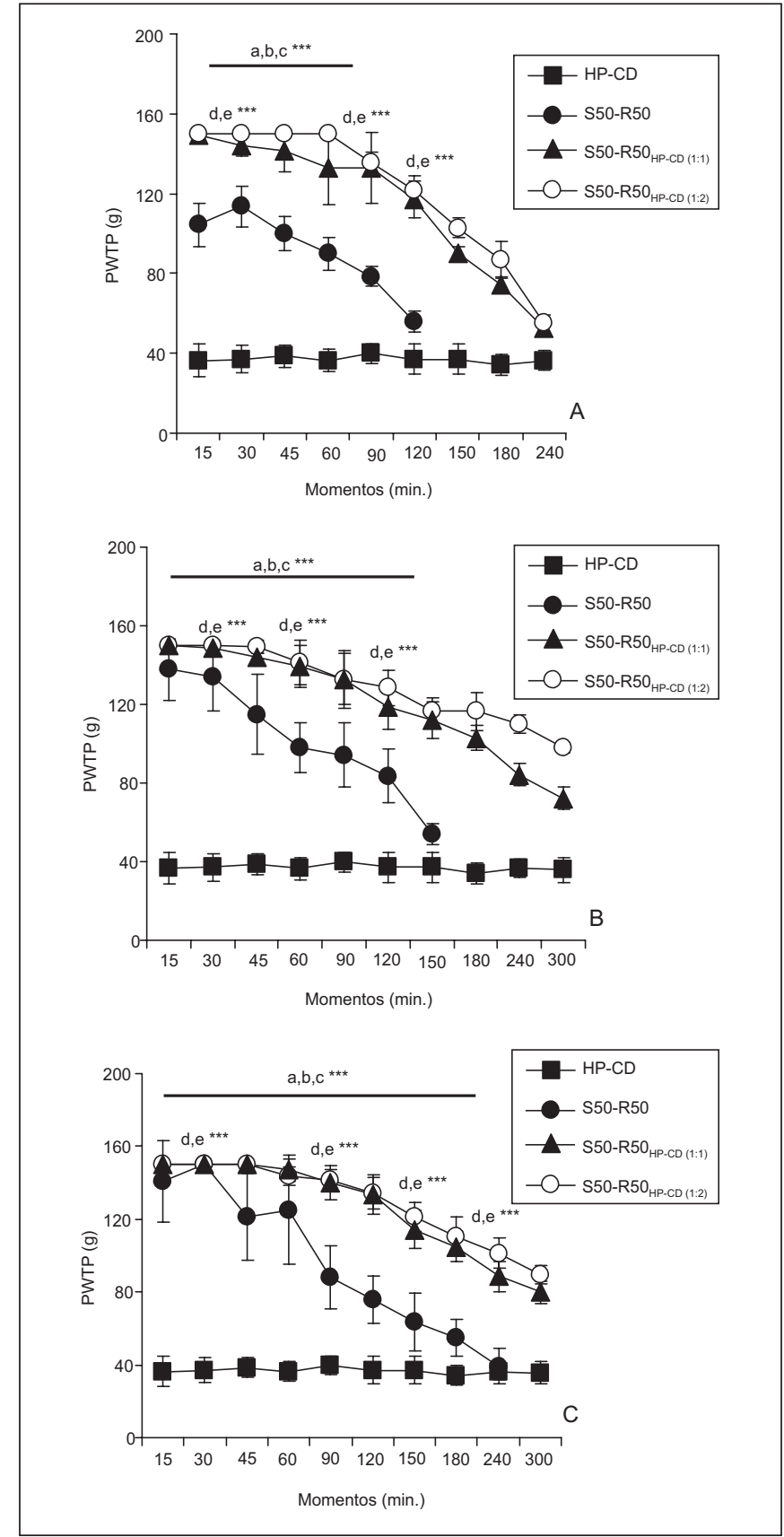

Figura 3 - Curvas Efeito versus Tempo no Teste PWTP para os Grupos

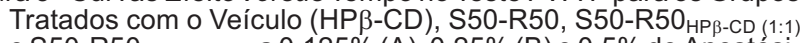
e S50-R50 $0_{\mathrm{HP} \beta-\mathrm{CD}(1: 2)}$ a $0,125 \%$ (A), $0,25 \%$ (B) e $0,5 \%$ de Anestésico $(C)$. Valores expressos em Média $\pm D P(n=7)$.

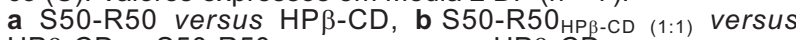

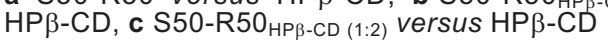

d S50-R50 ${ }_{H P \beta-C D ~(1.1)}$ versus S50-R50, e S50-R50 ${ }_{H P \beta-C D ~(1: 2)}$ versus S50-R50.

${ }^{*} p<0,05,{ }^{* *} p<0,01,{ }^{* * *} p<0,001$ (Análise de Variância) 

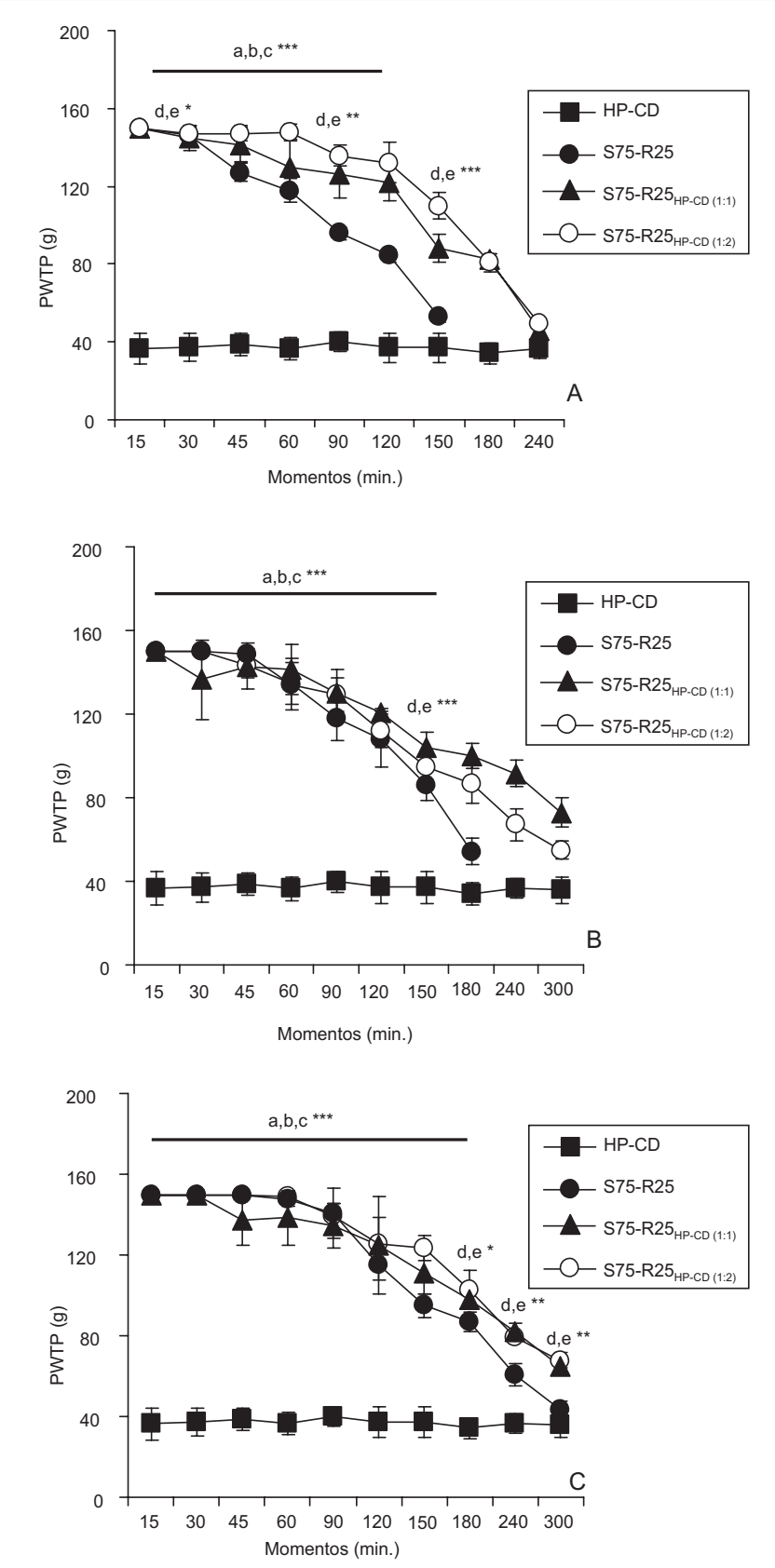

Figura 4 - Curvas Efeito versus Tempo no Teste PWTP para os Grupos Tratados com o Veículo (HP $\beta$-CD), S75-R25, S75-R25 HP $\beta$-CD (1:1) e S75-R25 HP $\beta-C D(1: 2)$ a $0,125 \%$ (A), $0,25 \%$ (B) e $0,5 \%(C)$. Valores expressos em Média \pm DP $(n=7)$. a S75-R25 versus HP $\beta-C D$, b S75-R25 HPß-CD (1:1) versus HP $\beta-C D$, c S75-R25 $5_{H P-C D}(1: 2)$ versus HP $\beta-C D$

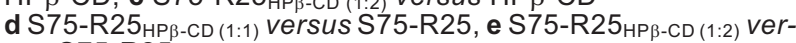
sus $\mathrm{S} 75-\mathrm{R} 25$.

${ }^{*} p<0,05,{ }^{* *} p<0,01,{ }^{* * *} p<0,001$ (Análise de Variância)

\section{DISCUSSÃO}

As CD podem ser descritas como cápsulas cilíndricas apresentando uma cavidade interna hidrofóbica que, uma vez preenchida com a molécula de uma substância de tamanho molecular e polaridade compatíveis, formam complexos de inclusão. Por uma série de conveniências (preço, disponibilidade, dimensões da cavidade) uma das CD naturais, a $\beta-C D$, tornou-se a mais amplamente utilizada para complexação e sistemas de liberação controlada de drogas. No entanto, sua baixa solubilidade aquosa e indução de nefrotoxicidade direcionaram as pesquisas para a síntese de CD mais solúveis, menos tóxicas e mais apropriadas para uso parenteral, como a hidroxipropil $\beta-C D^{7}$ (HP $\left.\beta-C D\right)$. Neste estudo, utilizou-se a HP $\beta$-CD com o objetivo de minimizar problemas quanto ao uso farmacêutico das $C D$, especialmente com relação à solubilidade aquosa e toxicidade sistêmica.

Estudos mostraram que a utilização de HP $\beta$-CD e de alguns outros derivados (CD sulfatadas) aumentou a solubilidade de AL como etidocaína, lidocaína, prilocaína, mepivacaína e, especialmente, bupivacaína ${ }^{19}$, bem como melhorou os índices terapêuticos dessas drogas ${ }^{8-11}$. De fato, os resultados apresentados neste trabalho mostraram aumento linear na solubilidade dos anestésicos bupivacaína S50-R50 e S75-R25, que foram favorecidos pela formação de complexos de inclusão com a HP $\beta$-CD. As características físico-químicas semelhantes da S50-R50 e S75-R25 direcionaram a complexação com HP $\beta-C D$, determinando valores de constantes de afinidade semelhantes e indicando um posicionamento favorável das drogas dentro da cavidade hidrofóbica da CD.

Com relação aos testes de eficácia anestésica, o bloqueio do nervo ciático foi utilizado por ser técnica bem descrita na literatura e modelo experimental apropriado para monitorar a eficácia de $A L$ em ratos ${ }^{20-21}$ e camundongos ${ }^{14-15,17}$.

O tratamento com as formulações livres de S50-R50 e S75-R25 (0,125\%; 0,25\% e 0,5\%) mostrou que a duração do bloqueio motor induzido por S75-R25 foi similar, mas de intensidade menor que o induzido por S50-R50. Aadministração de S75-R25 em camundongos potencializou a intensidade e prolongou o tempo de analgesia quando comparada à S50-R50, especialmente nas menores concentrações $(0,125 \%$ e $0,25 \%$ ). A literatura relata que a S75-R25 proporciona boas condições para a realização do ato anestésico-cirúrgico com adequados bloqueios motor e sensorial ${ }^{22-23}$ prolongando a analgesia pós-operatória em relação à $\mathrm{S} 50-\mathrm{R} 50^{24}$. Além disso, a S75-R25 mostrou-se formulação mais segura que a $\mathrm{S} 50-\mathrm{R} 50^{5,22-23}$, fato este que motivou a investigação com formulações de S75-R25 complexadas com HP $\beta-C D$.

Os resultados apresentados neste estudo mostraram que as formulações complexadas com HP $\beta$-CD reduziram de forma significativa a latência, sem modificar a intensidade do bloqueio motor ( $\left.E_{\text {máx }}\right)$, em relação às formulações livres. Outros parâmetros, como duração do bloqueio motor e efeito total do $\mathrm{AL}(A \cup C)$, só foram afetados pelos complexos na proporção molar 1:2 (AL:CD). Provavelmente, esses efeitos devem-se às propriedades da HP $\beta-C D$ como maior solubilidade em 
água, facilitando o acesso de anestésicos hidrofóbicos, como S50-R50 e S75-R25, ao local de ação.

Comparando-se as formulações livres e os sistemas com HP $\beta$-CD para os dois anestésicos, observou-se que houve benefício em relação à intensidade e duração da analgesia em todas as concentrações utilizadas, indicando potencialização do bloqueio nervoso diferencial devido à interação tanto da S50-R50 quanto da S75-R25 com a HP $\beta-C D$, aumentando o tempo de permanência dos AL no local de ação.

Modificações na proporção molar AL:CD mostraram que o tratamento com os complexos 1:2 prolongou o tempo de recuperação do bloqueio motor em relação às formulações livres nas concentrações utilizadas $(0,125 \% ; 0,25 \%$ e $0,5 \%$ para S50-R50 e 0,5\% para S75-R25), embora os complexos 1:1 e 1:2 não tenham sido diferentes entre si após comparações na qualidade e/ou duração do bloqueio sensorial. Essa alteração na farmacodinâmica dos AL pode ser um efeito inerente à $H P \beta-C D$ (já que foi observado tanto com S50-R50 quanto com S75-R25), devido a fatores como diferenças na razão de transferência das drogas ou dissociação do complexo em relação à $\mathrm{HP} \beta-C D^{8}$.

A liberação controlada de AL tem como objetivos principais melhorar o índice terapêutico das drogas para os tratamentos da dor aguda e utilização no período pós-operatório, reduzindo a toxicidade sistêmica e agindo mais sobre fibras sensoriais do que motoras ${ }^{8-11}$. Os efeitos analgésicos mais pronunciados obtidos após a complexação com HP $\beta-C D$ tornam as formulações S50-R50 $0_{H P \beta-C D}$ e S75-R2 $5_{H P \beta-C D}$ de grande interesse para o alívio da dor no período pós-operatório, com a vantagem de serem administradas em menor concentração que as preparações de AL comerciais. Entretanto, por ser uma formulação potencialmente menos tóxica, a mistura enantiomérica de bupivacaína (S75-R25) representa uma alternativa para o desenvolvimento de formulações de liberação controlada mais seguras e eficazes do que a bupivacaína (S50-R50).

\section{Drug-Delivery Systems for Racemic Bupivacaine (S50-R50) and Bupivacaine Enantiomeric Mixture (S75-R25): Cyclodextrins Complexation Effects on Sciatic Nerve Blockade in Mice}

Daniele Ribeiro de Araújo, M.D.; Leonardo Fernandes Fraceto, M.D.; Angélica de Fátima de Assunção Braga, TSA, M.D.; Eneida de Paula, PhD

\section{INTRODUCTION}

Bupivacaine is the amino-amide local anesthetic (LA) more widely used in surgical procedures, especially for prolonged regional anesthesia. Bupivacaine-induced side effects (central nervous system and cardiovascular toxicity) have led to the search for new agents with similar blockade profile, but with lower cardiac toxicity ${ }^{1}$. Results were LA such as ropivacaine and levobupivacaine, synthesized in the enantiomeric form S(-) with have shown that in addition to molecular changes, stereoselectivity is a major factor to decrease bupivacaine's cardiotoxicity. Recent studies have shown decreased arrhythmogenic ability and lower risk of levobupivacaine-induced seizures ${ }^{2-3}$, as well as the maintenance of anesthetic properties similar to bupivacaine ${ }^{4}$. Investigations on stereoselectivity have allowed for changes in bupivacaine stereoisomers $\mathrm{R}(+)$ and S(-) and for the synthesis of a new local anesthetic formulation with $25 \%$ $\mathrm{R}(+)$-bupivacaine and $75 \% \mathrm{~S}(-)$-bupivacaine, improving drug's anesthetic profile as compared to levobupivacaine and increasing its safety margin ${ }^{5}$.

Although there are differences in enantiomers pharmacokinetic and pharmacodynamic profiles, physico-chemical properties such as water or lipid solubility and $\mathrm{pKa}$ are preserved ${ }^{4}$, and they are factors potentially limiting therapeutic benefits of traditional systems. An alternative would be the development of drug delivery systems with LA in carriers such as cyclodextrins (CD) which allow the manipulation of some physico-chemical properties in addition to improving therapeutic effects, thus favoring their clinical use ${ }^{6}$.

CDs are cyclic oligosaccharides made of glucose units (Figure 1) able to complex molecules of adequate size and polarity in their hydrophobic cavity forming inclusion complexes. Complexation changes physico-chemical properties, such as drug solubility, stability and bioavailability ${ }^{7}$. In fact, this property makes CD very promising for LA complexation, and several studies with racemic bupivacaine have shown prolonged duration and significant therapeutic index increase $8-11$. So, it is interesting to evaluate whether complexation with $C D$ would also change the anesthetic profile of bupivacaine enantiomeric mixture, a potentially less toxic formulation, as compared to racemic bupivacaine. This study

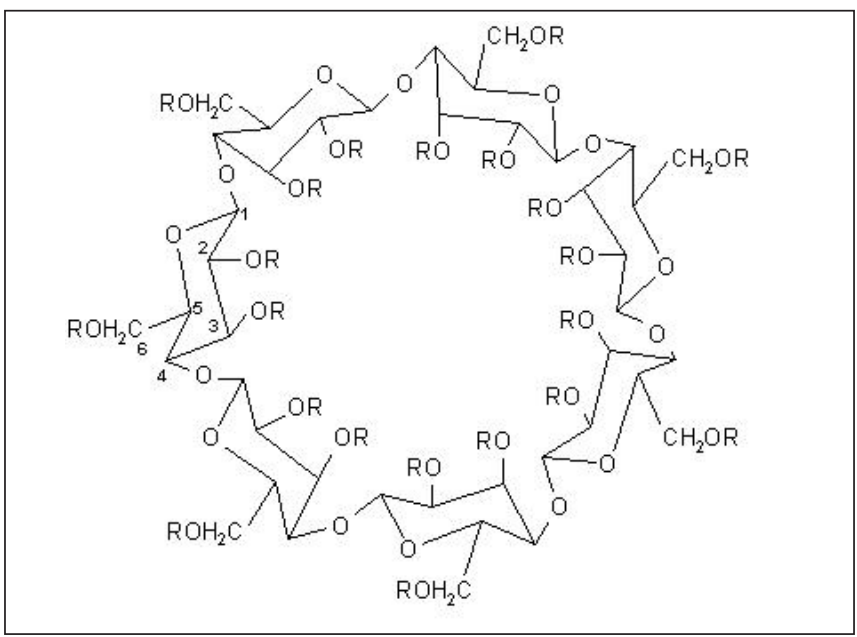

Figure 1 - Chemical $\beta-C D$ structure, with 7 Glucose Monomers and $\mathrm{R}=\mathrm{H}$. R $=\mathrm{CH}_{2} \mathrm{CHOHCH}_{3}$ or $\mathrm{H}$, for $\mathrm{HP} \beta-\mathrm{CD}$ (replacements in carbons 2, 3 and 5) 
aimed at preparing, characterizing and evaluating in mice the anesthetic efficacy of inclusion complexes of bupivacaine enantiomeric mixture (S75-R25) and racemic bupivacaine (S50-R50) with hydroxypropyl $\beta$-cyclodextrin (HP $\beta$-CD) comparing them to clinically available preparations.

\section{METHODS}

Solid complexes were prepared by mixing adequate amounts of HP $\beta-C D$ and S50-R50 or S75-R25 volumes in water to obtain molar ratios $1: 1$ or $1: 2$. The mixtures were kept shaking up during 24 hours at room temperature. Following, the solution was lyophilized and stored at $-20^{\circ} \mathrm{C}$. Solid complexes were weighed in sufficient amounts to obtain desired drug concentrations and then were dissolved in buffer $(20 \mathrm{mM}$ HEPES, pH 7.4, with $154 \mathrm{mM} \mathrm{NaCl}$ ). S50-R50 and S75-R25 concentrations were: $0.125 \% ; 0.25 \%$ e $0.5 \%$.

Phase solubility studies were performed ${ }^{12}$ to determine the affinity constants $(K)$ between HP $\beta-C D$, and each LA, for physico-chemical characterization of formed complexes. Experiments were carried out to evaluate the solubility of both LA in $\mathrm{pH} 7.4$ and - in the absence of HP $\beta-C D$ - both S50-R50 and S75-R25 are insoluble in the concentration used (30 $\mathrm{mM})$. In our study, increasing HP $\beta-C D$ concentrations $(0.5$, 10 and $20 \mathrm{mM}$ ) were added to $30 \mathrm{mM}$ of S50-R50 or S75-R25 and then dissolved in buffer solution (50 $\mathrm{m} \mathrm{M} \mathrm{HEPES,} \mathrm{pH} \mathrm{7.4,}$ with $154 \mathrm{mM} \mathrm{NaCl}$ ).

Samples were agitated at room temperature for 24 hours until balance was reached. Then, samples were filtered and analyzed by spectrophotometry (Beckman DU-70 spectrophotometer) at a wavelength of $260 \mathrm{~nm}^{13}$, and LA concentration in the solution was calculated. All experiments were repeated three times. High molar ratio LA: HP $\beta$-CD (until 2:1) used in this experiment is justified by the fact that the affinity constant $(K)$ is calculated using the linear portion of phase solubility diagram, according to the following equation ${ }^{12}$ :

$$
K=\frac{\text { Slope }}{\text { Initial LA Solubility }(1-\text { slope })}
$$

\section{Anesthetic Efficacy Evaluation}

This experiment was performed after the approval by the Animal Experiment Ethics Committee, Instituto de Biologia, Universidade Estadual de Campinas (CEEA) - IB UNICAMP, which follows the standards of the Brazilian College of Animal Experiment (COBEA). The study involved groups of 7 male albino mice of Swiss strain, with approximately 60 days of age and body weight varying from 30 to 35 grams. Animals supplied by the Lab Animals Facility, UNICAMP (CEMIB) were submitted to 12-hour dark/light cycle with free water and food, and were collectively lodged (5 animals per cage) and adapted to experiment site for at least 7 days.
Anesthetic efficacy experiments were performed by sciatic nerve block in mice ${ }^{14}$. Formulations, including vehicle, were randomly administered by $0.1 \mathrm{~mL}$ infiltration in the popliteal space located on the posterior knee close to sciatic nerve. Motor and sensory blocks were simultaneously evaluated and all measures were obtained by the same investigator. Animals were observed for 24 hours after treatment to check the incidence of systemic (seizures and mortality) or local (failures in recovering normal movements of the injected paw) toxic effects. Experimental groups were distributed as follows:

- Control Group: HP $\beta-C D$

- Group S50-R50: racemic bupivacaine

- Group S75-R25: bupivacaine enantiomeric mixture

- Group S50-R50 HP -CD (1:1): racemic bupivacaine with hydroxypropyl $\beta$-cyclodextrin in $1: 1$ molar ratio (S50-R50:HP $\beta-C D)$.

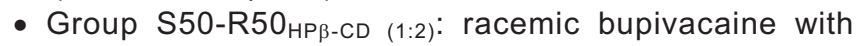
hydroxypropyl $\beta$-cyclodextrin in $1: 1$ molar ratio (S50-R50:HP $\beta-C D)$

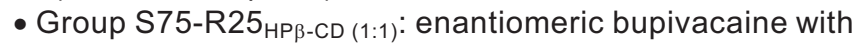
hydroxypropyl $\beta$-cyclodextrin in $1: 1$ molar ratio (S75-R25:HP $\beta-C D)$

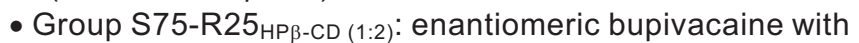
hydroxypropyl $\beta$-cyclodextrin in 1:2 molar ratio (S75-R25:HP $\beta-C D)$

The ability of each mouse to normally walk with the four paws on a metal mesh (with $5 \mathrm{~mm}$ diameter holes) in normal and inverted position was measured before the experiment to evaluate motor block. Only animals fulfilling this requirement were submitted to the experiment. Motor block level was evaluated according to score values 0 (normal movements), 1 (inability to totally bend injected limb) and 2 (total injected limb paralysis) in the following moments: every minute during the first 5 minutes after administration and then in 10-minute intervals until total movements recovery ${ }^{15}$. Onset (time elapsed between injection and motor block 1 or 2 ), time to reach highest score $\left(\mathrm{T}_{\max }\right)$, highest score value (maximum effect $\left.E_{\max }\right)$, time to motor function recovery and total local anesthetic effect (estimated by the area under the effect-time curve, AUC) were evaluated.

Sensory block was measured by mechanical stimulation ${ }^{16}$ with adequate equipment called analgesimeter, which generates gradual strength increase (in grams) on the dorsal surface of animal's paw. To prevent stress-induced analgesia, each animal was involved in a towel so that head and limbs with free. Paw withdrawal reflex was considered representative of pain threshold (Pain Withdrawal Threshold to Pressure - PWTP).

PWTP baseline was measured before experiments and animals presenting pain thresholds below or above 30 to $50 \mathrm{~g}$ were excluded from the experiment. Maximum cut-off pressure value of $150 \mathrm{~g}$ was considered representative of sensory block. After vehicle or drug administration, animals were measured for 5 hours at 15-minute intervals in the first hour, 30-minute intervals in the second and third hours and at 
60 -minute intervals for the last two hours. Analgesia was defined as increased tolerated pressure threshold of at least $50 \%$ above the group treated with vehicle. Analgesic end point was established as the absence of statistical difference between test and control groups ${ }^{17}$.

Kruskal-Wallis test was used for statistical analysis of motor block (onset, $\mathrm{T}_{\max }$, recovery time, $\mathrm{E}_{\max }$ and $\mathrm{AUC}$ ) and data were expressed in median (minimum limit and maximum limit). PWTP values during sensory block were expressed in mean \pm standard deviation (SD) and were evaluated in individual times by Analysis of Variance (One-way ANOVA) with further Tukey-Kramer test. Statistical significance was defined as $p<0.05^{18}$.

\section{RESULTS}

Characterization of Inclusion Complexes with Cyclodextrins

Complexation of S50-R50 and S75-R25 with HP $\beta-C D$ at $\mathrm{pH}$ 7.4 and $25^{\circ} \mathrm{C}$ could be observed by phase solubility experiments (Figure 2). Systems phase solubility diagram indicates formation of soluble complexes with linear increase in solubility of S50-R50 and S75-R25 (approximately 1.3-fold) as a function of tested HP $\beta-C D$ concentrations. Values obtained for affinity constants $(\mathrm{K})$ were similar for both $\mathrm{LA}$, being 14.7 $\mathrm{M}^{-1}$ for S50-R50:HP- $\beta$ CD and $14.3 \mathrm{M}^{-1}$ for S75-R25:HP- $\beta C D$.

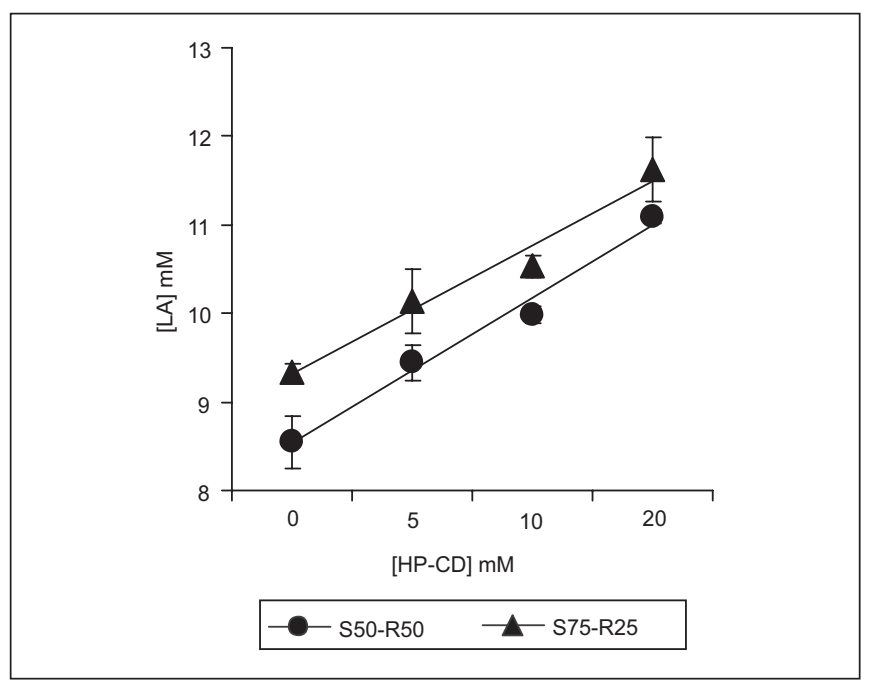

Figure 2 - Solubility Curves for S50-R50 and S75-R25 in the Presence of Increasing HP- $\beta C D$ Concentrations, 50 mM HEPES buffer, $\mathrm{pH} 7.4,25^{\circ} \mathrm{C}$

\section{Motor Block Evaluation}

Plain and complexed LAinjection has changed, in concentration-dependent manner, the motor function after sciatic nerve block with reversible loss of motor reflexes in all treated animals (Table I). The group treated with vehicle (HP- $\beta C D)$ has not shown any sign of motor block.
Comparisons between plain drugs (S50-R50 and S75-R25) have shown that S75-R25 has induced shorter onset ( $p<$ 0.001 ) in all tested concentrations, although lower motor block intensity has been observed in $0.125 \%$ concentration $\left(p<0.001\right.$ as shown by $E_{\text {max }}$ values) as compared to S50-R50. However, motor block recovery time and total effect (AUC) induced by both commercial preparations were similar.

Complexed formulations with S50-R50 have induced shorter motor block onset as compared to plain drug in all tested concentrations ( $p<0.01$ for inclusion complexes $1: 1$ and 1:2). In addition, statistical analysis has also revealed differences between R50 HPB-CD (1:2) and S50-R50 for $T_{\max }(p<0.05)$, recovery time and AUC $(p<0.001$ and $p<0.05$, respectively) in $0.5 \%$ concentration.

With systems S75-R25 HP - CD (1:1 e 1:2), onset was significantly shorter ( $p<0.05$ and $p<0.01$, respectively) as compared to plain formulation in different concentrations. AUC was significantly higher $(p<0.05)$ for the complex in $1: 2$ molar ratio as compared to $1: 1$ complex and plain formulation in $0.5 \%$ concentration.

\section{Sensory Block Evaluation}

Sensory block evaluation by PWTP test has shown concentration-dependent ratios for plain formulations and inclusion complexes of S50-R50 or S75-R25 with HP $\beta-C D$. Effects of treatment with $\mathrm{HP} \beta-\mathrm{CD}$, plain and complexed drugs were evaluated along time (Figures 3 and 4 ).

Vehicle infiltration in the sciatic nerve has not changed baseline values for pain threshold in mice. However, all formulations have increased treated animals pain threshold, which was statistically different as compared to control group $(p<$ 0.001 ). In addition, complexes with HP $\beta-C D$ have increased sensory block duration and intensity as compared to plain drugs ( $p<0.001)$. However comparisons between complexes (1:1 and 1:2, in all concentrations) have shown similar sensory block profiles.

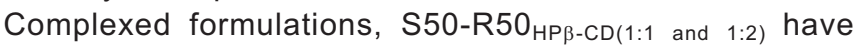
changed animals response to mechanical stimulation at PWTP, increasing pain threshold and nociceptive effect duration. Complexes in $0.125 \%$ concentration have induced longer analgesia ( up to 4 hours) and effect intensity (approximately 2 -fold at 120 minutes, $p<0.001$ ) as compared to plain formulation (Figure $3 \mathrm{~A}$ ). Complexed formulations in $0.25 \%$ concentration have also improved analgesic effect at 150 minutes as compared to S50-R50 (2-fold, p < 0.001) (Figure $3 \mathrm{~B})$. Complexed formulations at $0.5 \%$ have shown similar profile as intermediate concentration, being the analgesic intensity significantly higher $(p<0.001)$ as compared to plain drug (Figure 3C).

In groups treated with complexes S75-R25 $5_{\mathrm{HP} \beta-\mathrm{CD}(1: 1 \mathrm{e} \text { 1:2) }}$ in different concentrations, analgesic intensity was approximately 1.5 to 1.8 -fold higher with complexed drugs $(p<0.001$ in $0.125 \%$ and $0.25 \%$ concentrations) as compared to plain drug (240 to 300 minutes $x 150$ to 300 minutes, respectively) (Figures $4 \mathrm{~A}$ and $4 \mathrm{~B}$ ). In $0.5 \%$ concentration, although analge- 
Table I - Onset, $\mathrm{T}_{\max }$, Recovery Time, $\mathrm{E}_{\max }$ and Total Motor Block Effect (AUC) Induced by Formulations S50-R50 or S75-R25 Plain and Complexed with $\mathrm{HP} \beta-\mathrm{CD}(1: 1$ and $1: 2)$ during Sciatic Nerve Block in Mice

\begin{tabular}{|c|c|c|c|c|c|c|}
\hline $\begin{array}{l}\text { Concentration } \\
(\%)\end{array}$ & Groups & $\begin{array}{l}\text { Onset } \\
\text { (sec.) }\end{array}$ & $\begin{array}{c}\mathrm{T}_{\max } \\
\text { (min.) }\end{array}$ & $\begin{array}{l}\text { Recovery Time } \\
\text { (min.) }\end{array}$ & $\begin{array}{c}E_{\max } \\
\text { (score) }\end{array}$ & $\begin{array}{c}\text { AUC } \\
\text { (score/h) }\end{array}$ \\
\hline \multirow[t]{6}{*}{$0.125 \%$} & S50-R50 & $60(60-120)$ & $3(2-3)$ & $20(-)$ & $2(-)$ & $22.5(19-28)$ \\
\hline & 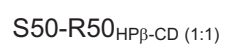 & $30(30-60)^{\mathrm{a}^{*+*}}$ & $1(1-2)$ & $30(20-40)$ & $1(1-2)$ & $24(17-36)$ \\
\hline & S50-R50 HP -CD (1:2) & $25(15-35)^{\mathrm{b}^{* *}}$ & $1(1-4)$ & $40(30-50)^{b^{*}}$ & $1(1-2)$ & $34(24-48)$ \\
\hline & S75-R25 & $40(38-46)^{f+x t}$ & $1(-)$ & $30(30-50)$ & $1(-)^{\mathrm{f} * \star \star * t}$ & $34(24-44)$ \\
\hline & S75-R25 HP & $30(25-40)^{c^{*}}$ & $1(1-3)$ & $40(20-60)$ & $1(-)$ & $34(24-54)$ \\
\hline & S75-R25 HP -CD (1:2) & $20(18-25)^{\mathrm{d}^{+x}}$ & $2(1-2)$ & $40(30-60)$ & $1(1-2)$ & $47.5(27-54)$ \\
\hline \multirow[t]{6}{*}{$0.25 \%$} & S50-R50 & $60(60-120)$ & $2(2-3)$ & $40(30-40)$ & $2(-)$ & $41(37-58)$ \\
\hline & S50-R50 ${ }_{H P \beta-C D ~(1: 1)}$ & $30(30-60)^{\mathrm{a}^{*+*}}$ & $1(1-2)$ & $50(40-60)$ & $2(1-2)$ & $60(44-78)$ \\
\hline & S50-R50 HP -CD (1:2) & $20(20-30)^{\mathrm{b} * *}$ & $2(1-3)$ & $70(50-80)^{\mathrm{b}^{*}}$ & $2(1-2)$ & $67(44-80)^{b^{*}}$ \\
\hline & S75-R25 & $40(38-46)^{p+p+x}$ & $1(-)$ & $30(30-50)$ & $1(-)^{f+x+x}$ & $34(24-44)$ \\
\hline & S75-R25 & $25(20-30)^{c^{*}}$ & $1(-)$ & $60(30-60)$ & $1(1-2)$ & $54(24-54)$ \\
\hline & S75-R25 HP -CD (1:2) & $18(17-22)^{\mathrm{d}^{\star \star}}$ & $2(1-4)$ & $70(40-80)$ & $2(1-2)$ & $74(48-78)$ \\
\hline \multirow[t]{6}{*}{$0.5 \%$} & S50-R50 & $60(-)$ & $1(-)$ & $50(30-60)$ & $2(-)$ & $58(50-88)$ \\
\hline & S50-R50 HP -CD (1:1) & $30(-)^{\mathrm{a}^{\star \star}}$ & $1(1-2)$ & $60(50-70)$ & $2(-)$ & $88(78-98)$ \\
\hline & 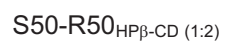 & $15(10-17)^{\mathrm{b}^{\mathrm{b} *}}$ & $2(1-3)^{b^{*}}$ & $90(80-100)^{b^{b+4 t}}$ & $2(-)$ & $100.5(97.5-108)^{b^{*}}$ \\
\hline & S75-R525 & $40(35-45)^{p+p+c}$ & $1(1-3)$ & $60(40-60)$ & $2(-)$ & $67.5(50.5-68)$ \\
\hline & S75-R25 HP & $17(15-25)^{\mathrm{c}^{\star \star}}$ & $1(1-3)$ & $60(50-60)$ & $2(1-2)$ & $60(49-68)$ \\
\hline & S75-R25 HP -CD (1:2) & $12(10-12)^{\mathrm{d}^{* t}}$ & $2(1-2)$ & $90(60-100)^{\mathrm{d}^{*}}$ & $2(1-2)$ & $97.5(68-118)^{\text {d.e } e^{*}}$ \\
\hline
\end{tabular}

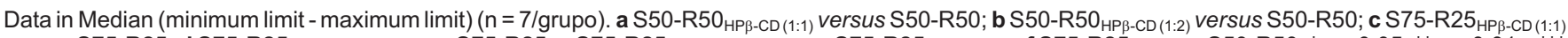

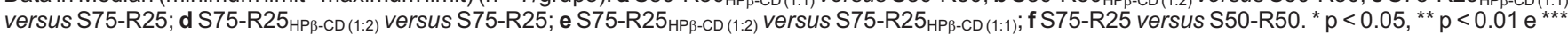
$\mathrm{p}<0.001$ (Kruskal-Wallis Test)

sic duration with complexes has been similar to plain drug, post-complexes pain threshold was significantly higher $(p<$ 0.01) (Figure 4C).

\section{DISCUSSION}

CD may be described as cylindrical capsules with internal hydrophobic cavity which, once filled with a molecule of compatible molecular size and polarity, generate inclusion complexes. For several conveniences (price, availability, cavity size) $\beta-C D$ has become the most widely used CD for complexation and release systems of drugs. However, its low water solubility and nephrotoxicity have directed research to the synthesis of more soluble, less toxic and more adequate $C D$ for parenteral use, such as hydroxypropil $\beta-C D{ }^{7}$ (HP $\beta-C D)$. Our study has used HP $\beta-C D$ aiming at minimizing problems of $C D$ pharmaceutical use, especially low water solubility and systemic toxicity.

Studies have shown that HP $\beta-C D$ and some other CD derivatives (sulfated CD), have increased the solubility of LA such as etidocaine, lidocaine, prilocaine, mepivacaine and especially bupivacaine ${ }^{19}$, in addition to improving their therapeutic index ${ }^{8-11}$. In fact, this study has shown linear increase in S50-R50 and S75-R25 solubility, which were favored by inclusion complexes formation with HP $\beta-C D$. Similar physico-chemical characteristics of S50-R50 and S75-R25 have oriented complexation with $\mathrm{HP} \beta-\mathrm{CD}$ determining similar affinity constant values and indicating favorable drug positioning in CD hydrophobic cavity.

The sciatic nerve blockade in mice was choosed because it is a well described experimental model to study $L^{14,15,17,20,21}$. Treatment with plain S50-R50 and S75-R25 (0.125\%; $0.25 \%$ and $0.5 \%$ ) formulations has shown that motor block induced by S75-R25 was similar but lower in intensity as compared to S50-R50. The administration of S75-R25 in mice has increased intensity and prolonged analgesia as compared to S50-R50, especially in lower concentrations $(0.125 \%$ and $0.25 \%$ ). Literature reports that S75-R25 provides satisfactory conditions for surgical procedures with adequate motor and sensory blocks ${ }^{22-23}$, prolonging postoperative analgesia as compared to S50-R50 ${ }^{24}$. In addition, S75-R25 has shown to be safer than $\mathrm{S} 50-\mathrm{R} 50^{5,22-23}$, which has motivated investigations with complexed S75-R25 HP $\beta-C D$ formulations. Our results have shown that complexed formulations with HP $\beta$-CD have significantly shortened onset without changing motor block intensity $\left(E_{\max }\right)$ as compared to plain formulations. Other parameters, such as motor block duration and total LA effect (AUC) were only affected by complexes in 1:2 molar ratio (LA:CD). These effects were probably due to HP $\beta$-CD properties, such as higher water solubility, helping 


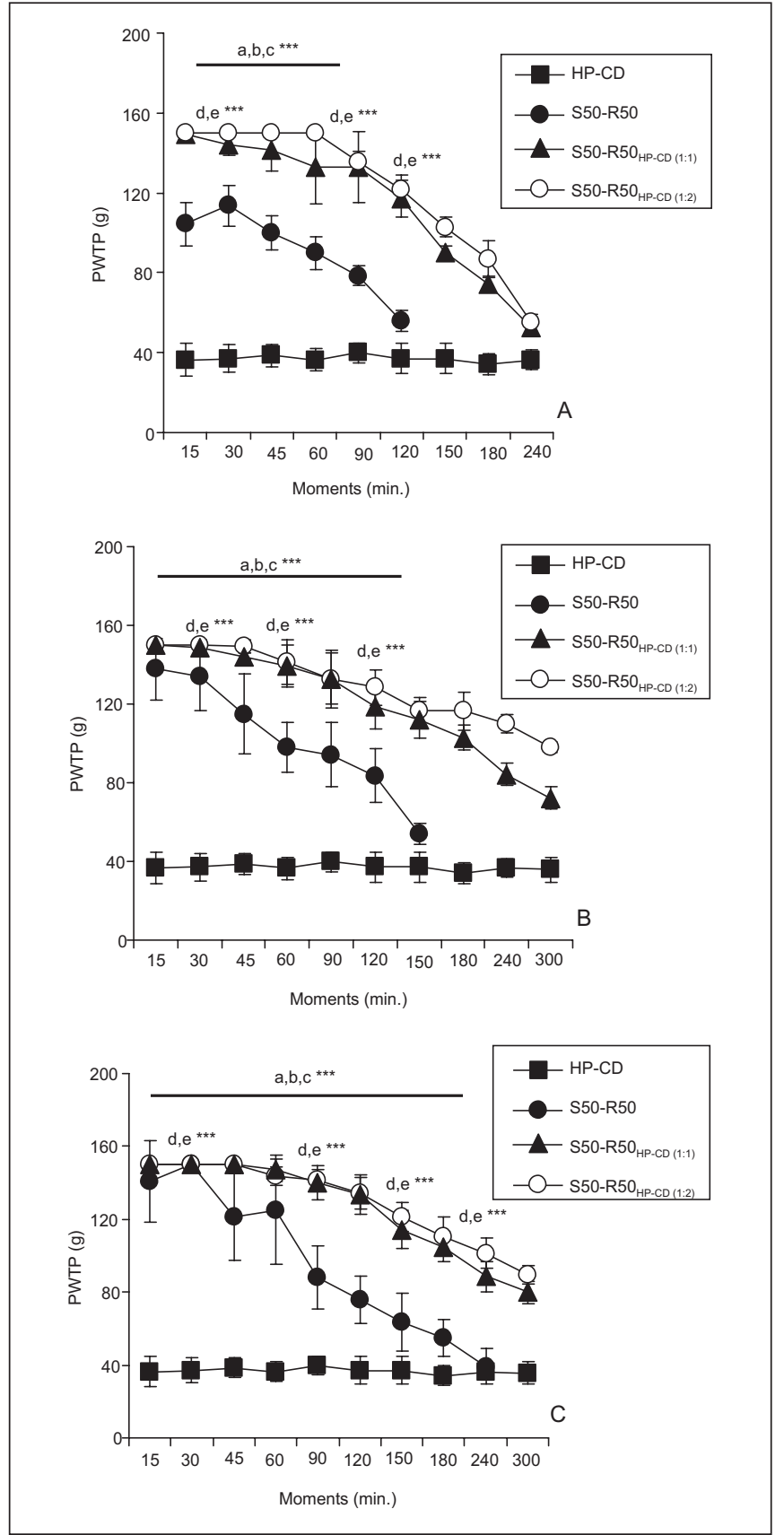

Figure 3 - Effect versus Time Curves in PWTP test for Groups treated with vehicle (HP $\beta-C D)$, S50-R50, S50-R50 $\mathrm{HP \beta}-\mathrm{CD}(1: 1)$ and $\mathrm{S} 50-\mathrm{R}_{5} 0_{\mathrm{HP}-\mathrm{CD}(1: 2)}$ at $0.125 \%(\mathrm{~A}), 0.25 \%(\mathrm{~B})$ and $0.5 \%$ Concentrations $(\mathrm{C})$

Values in Mean \pm SD $(n=7)$. a S50-R50 versus HP $\beta$-CD, b S50-R50 $0_{H P \beta-C D ~(1: 1)}$ versus HP $\beta-C D, c$ S $50-R 50_{H P \beta-C D ~(1: 2)}$ versus HP $\beta-C D$ d S50-R50HP -CD (1:1) versus S50-R50, e S50-R50HPß-CD (1:2) versus S50-R50. * $p<0.05,{ }^{* *} p<0.01$ *** $p<0.001$ (Analysis of Variance)

the access of hydrophobic anesthetics such as S50-R50 and S75-R25, to the site of action.

In comparing plain formulations and systems with HP $\beta-C D$ for both anesthetics, it has been observed a benefit in analgesia intensity and duration in all tested concentrations, indicat-

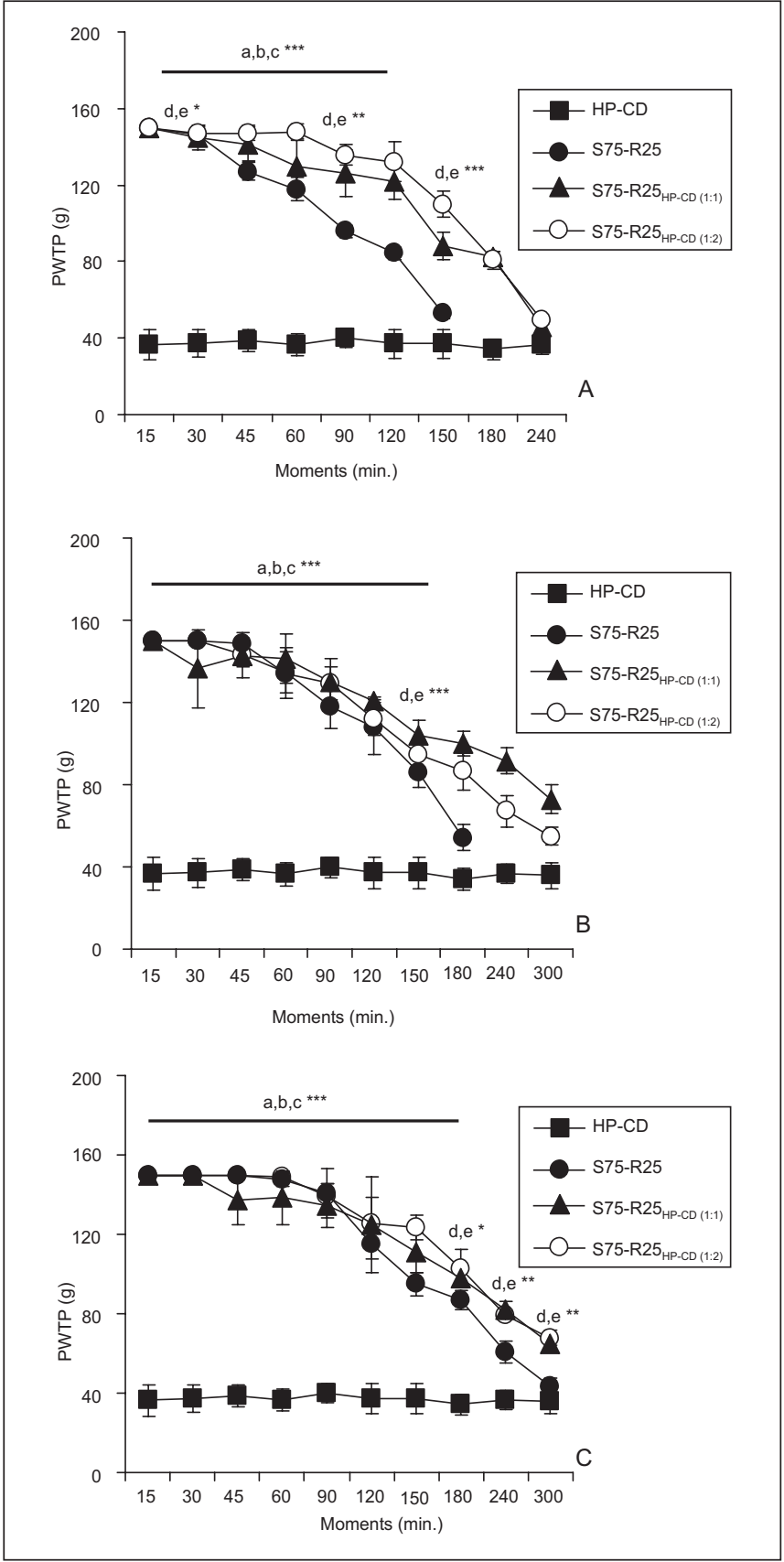

Figure 4 - Effect versus Time Curves in PWTP test for groups trea-

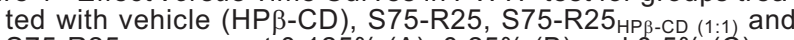
S75-R25 $5_{\text {HP-CD (1:2) }}$ at $0,125 \%(\mathrm{~A}), 0.25 \%(\mathrm{~B})$ and $0.5 \%$ (C) Values in Mean \pm SD $(n=7)$. a S75-R25 versus HP $\beta-C D, b$ S75-R25HP-CD (1.1) versus HP $\beta-C D$, c S75-R25 HP $\beta-C D(1.2)$ versus HP $\beta-C D$ d S $75-R 25_{H P \beta-C D}$, 1:1) versus S $75-R 25$, e S75-R25 HPß-CD (1:2) versus S75-R25. * $p<0.05,{ }^{* *} p<0.01$, ${ }_{* * *} p<0.001$ (Analysis of Variance)

ing differential nervous block potentiation due to interaction both of S50-R50 and S75-R25 with HP $\beta-C D$, thus increasing permanence time of LA in the site of action.

Changes in LA:CD molar ratio have shown that 1:2 complexes have prolonged motor block recovery time as com- 
pared to plain formulations in all concentrations $(0.125 \%$; $0.25 \%$ and $0.5 \%$ for S50-R50 and $0.5 \%$ for S75-R25), although $1: 1$ and 1:2 complexes were not different between them after comparing sensory block quality and/or duration. This LA pharmacodynamic change may be an inherent HP $\beta$-CD effect (since it was observed both with S50-R50 and S75-R25), due to factors such as differences in drug transfer ratio or complex dissociation in relation to $\mathrm{HP} \beta-C D^{8}$.

LArelease systems aim primarily at improving drug therapeutic index to treat acute postoperative pain, decreasing systemic toxicity and acting more on sensory than on motor fibers ${ }^{8-11}$. More pronounced analgesic effects obtained after complexation with HP $\beta-C D$ make S50-R50 $0_{H P-C D}$ and S75-R25 $5_{H P \beta-C D}$ of great interest to relieve postoperative pain, with the advantage of being administered in lower concentrations as compared to commercially available LA. However, for being potentially less toxic, enantiomeric bupivacaine mixture (S75-R25) is an alternative for the development of more effective and safer drug release systems as compared to bupivacaine (S50-R50).

\section{REFERÊNCIAS - REFERENCES}

01. Mather LE, Chang DH - Cardiotoxicity with modern local anesthetics: is there a safer choice? Drugs, 2001;61:333-342.

02. Ohmura S, Kawada M, Ohta T et al - Systemic toxicity and resuscitation in bupivacaine, levobupivacaine, or ropivacaine-infused rats. Anesth Analg, 2001;93:743-748.

03. Santos AC, DeArmas PI - Systemic toxicity of levobupivacaine, bupivacaine and ropivacaine during continuous intravenous infusion to nonpregnant and pregnant ewes. Anesthesiology, 2001;95:1256-1264.

04 . Whiteside JB, Wildsmith JA - Developments in local anesthesia drugs. Br J Anaesth, 2001;87: 27-35.

05. Simonetti MPB, Batista RA, Ferreira FMC - Estereoisomeria: a interface da tecnologia industrial de medicamentos e da racionalização terapêutica. Rev Bras Anestesiol, 1998;48:390-399.

06. Araujo DR, Pinto LMA, Braga AFA et al - Formulações de anestésicos locais de liberação controlada: aplicações terapêuticas. Rev Bras Anestesiol, 2003;53:663-671.

07. Szejtli J - Medicinal applications of cyclodextrins. Med Res Rev, 1994;14:353-386.

08. Freville JC, Dollo G, Le Corre P et al - Controlled systemic absorption and increased anesthetic effect of bupivacaine following epidural administration of bupivacaine-hydroxypropylbeta-cyclodextrin complex. Pharm Res, 1996;13:1576-1580.

09. Dollo G, Thompson DO, Le Corre P et al - Inclusion complexation of amide-type local anesthetics with $\beta$-cyclodextrin and derivates. III. Biopharmaceutics of bupivacaine-SBE7- $\beta-C D$ complex following percutaneous sciatic nerve administration in rabbits. Int J Pharm, 1998;164:11-19.

10. Dollo G, Le Corre P, Freville JC et al - Biopharmaceutics of local anesthetic-cyclodextrin complexes following loco-regional administration. Ann Pharm Fr, 2000;58:425-432.

11. Estebe JP, Ecoffey C, Dollo G et al - Bupivacaine pharmacokinetics and motor blockade following epidural administration of the bupivacaine- sulphobutylether -7-beta-cyclodextrin complex in sheep. Eur J Anaesthesiol, 2002;19:308-310.

Revista Brasileira de Anestesiologia

Vol. 55, № 3, Maio - Junho, 2005
12. Higuchi T, Connors KA - Phase-solubility techniques. Adv Anal Chem Instr, 1965;4:117-212.

13. de Paula E, Schreier S - Use of a novel method for determination of partition coefficients to compare the effect of local anesthetics on membrane structure. Biochim Biophys Acta, 1995;1240: 25-33.

14. Leszczynska K, Kau ST - A sciatic nerve blockade method to differentiate drug-induced local anesthesia from neuromuscular blockade in mice. J Pharmacol Toxicol Methods, 1992;27:85-93.

15. Gantenbein $M$, Abat $C$, Attolini $L$ et al - Ketamine effects on bupivacaine local anaesthetic activity and pharmacokinetics of bupivacaine in mice. Life Sci, 1997;61:2027-2033.

16. Randall LO, Selitto JJ - A method for measurement of analgesic activity of inflamed tissue. Arch Int Pharmacodyn, 1957;111:409-419.

17. de Araujo DR, Cereda CM, Brunetto GB et al - Encapsulation of mepivacaine prolongs the analgesia provided by sciatic nerve blockade in mice. Can J Anaesth, 2004;51:566-572.

18. Zar JH - Biostatistical Analysis, $3^{\text {rd }}$ Ed, New Jersey, Prentice-Hall, 1996;180-216.

19. Dollo G, Le Corre P, Chevanne F et al - Inclusion complexation of amide-type local anesthetics with $\beta$-cyclodextrin and derivates. II. Evaluation of affinity constants and in vitro transfer rate constants. Int J Pharm, 1996;136:165-174.

20. Simonetti MPB, Valinetti EA, Ferreira FM - Avaliação da atividade anestésica local da S(-) bupivacaína: estudo experimental in vivo em nervo ciático de rato. Rev Bras Anestesiol, 1997;47:425-434.

21. Sinnott CJ, Strichartz GR - Levobupivacaine versus ropivacaine for sciatic nerve block in the rat. Reg Anesth Pain Med, 2003;28:294-303.

22. Tanaka PP, Souza RO, Salvalaggio MFO et al - Estudo comparativo entre a bupivacaína a $0,5 \%$ e a mistura enantiomérica de bupivacaína (S75-R25) a 0,5\% em anestesia peridural em pacientes submetidos a cirurgia ortopédica de membros inferiores. Rev Bras Anestesiol, 2003;53:331-337.

23. Cortes CAF, Oliveira AS, Castro LFL et al - Estudo comparativo entre bupivacaína a $0,5 \%$, mistura enantiomérica de bupivacaína (S75-R25) a $0,5 \%$ e ropivacaína a $0,75 \%$ associadas ao fentanil em anestesia peridural para cesarianas. Rev Bras Anestesiol, 2003;53:177-187.

24. Gonçalves RF, Lauretti GR, Mattos AL - Estudo comparativo entre bupivacaína a 0,5\% e mistura enantiomérica de bupivacaína (S75-R25) a 0,5\% em anestesia peridural. Rev Bras Anestesiol, 2003;53:169-176.

\section{RESUMEN}

Araujo DR, Fraceto LF, Braga AFA, Paula E - Sistemas de Liberación Controlada con Bupivacaína Racémica (S50-R50) y Mescla Enantiomérica de Bupivacaína (S75-R25): Efectos de la Complexación con Ciclodextrinas en el Bloqueo del Nervio Ciático en Ratones

JUSTIFICATIVA Y OBJETIVOS: Los efectos adversos asociados al uso de bupivacaína llevaron a la búsqueda por nuevos anestésicos locales ( $A L$ ) con perfil de bloqueo semejante y menos tóxicos, surgiendo nuevas preparaciones como la mezcla enantiomérica de bupivacaína (S75-R25). Los sistemas de liberación controlada, conteniendo $A L$ en carreadores como ciclodextrinas (CD), tienen como objetivo mejorar la eficacia anestésica y el índice terapéutico de esas drogas. Este estudio visó la preparación, caracterización y 
evaluación de la eficacia anestésica de los complejos de inclusión de la mezcla enantiomérica de la bupivacaína (S75-R25) y de la bupivacaína racémica (S50-R50) con

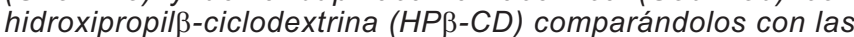
preparaciones actualmente utilizadas en la clínica.

MÉTODO: Los complejos de inclusión fueron preparados mezclándose cantidades apropiadas de HP $\beta-C D$ y S50-R50 ó S75-R25 en las razones molares (1:1 y 1:2) y caracterizados por estudios de solubilidad de fases. Se determinaron las constantes de afinidad $(K)$ de cada $A L$ por la HP $\beta-C D$. Los bloqueos motor y sensorial inducidos por las drogas libres $y$ complejadas fueron evaluados en ratones, a través del bloqueo del nervio ciático. Para la realización de los experimentos, se utilizaron tres concentraciones de AL: 0,125; 0,25 y 0,5\%.

RESULTADOS: Los estudios de solubilidad indicaron la formación de complejos de inclusión de S50-R50 y S75-R25 con $H P \beta-C D$, con valores de constante de afinidad $(K)$ análogos para los dos anestésicos: 14,7 $\mathrm{M}^{-1}$ (S50-R50: $H P-\beta C D)$ y 14,3 $\mathrm{M}^{-1}$ (S75-R25: HP- $\left.\beta C D\right)$. Las pruebas en animales mostraron que la complejidad potenció el bloqueo nervioso diferencial inducido por los $A L$ : i) la duración del bloqueo motor inducido por S75-R25 fue análogo al del
S50-R50, pero menos intenso $(p<0,001)$. Ya los complejos, $S 50-R 50 H P \beta-C D$ y S75-R25 HP - $C D$ redujeron la latencia $(p<$ 0,01 y $p<0,05$, respectivamente) sin modificar la intensidad del bloqueo motor ( $\left.E_{\text {máx }}\right)$, con relación a las drogas libres; ii) la evaluación del bloqueo sensorial mostró un aumento en la intensidad de la analgesia con los sistemas S50-R50 HP $\beta-C D$ (2 veces, $p<0,001)$ y S75-R25 HP $\beta-C D(1,5-1,8$ veces; $p<0,01$ y $p<0,001$, respectivamente), en las dos proporciones molares (1:1 y 1:2, AL:CD), además del prolongamiento de la duración del efecto analgésico, cuando comparados a las formulaciones S50-R50 y S75-R25.

CONCLUSIONES: Los efectos analgésicos más pronunciados logrados después de la complexación con HPß-CD muestran que ambas formulaciones, S50-R50 HP $\beta-C D$ y S75-R25 $H P \beta-C D$, son de grande interés para el alivio del dolor en el período postoperatorio, con la ventaja de ser administradas en menores concentraciones. Mientras, hay que resaltar que la mezcla enantiomérica de bupivacaína (S75-R25), por ser una droga menos tóxica, representa una alternativa en el desarrollo de formulaciones de liberación controlada más seguras y eficaces de que con la bupivacaína racémica (S50-R50). 